\title{
Instrumentation of the Very Forward Region at Future Linear Colliders - design and R\&D by the FCAL Collaboration
}

\author{
Iftach Sadeh* ${ }^{* \dagger}$ \\ School of Physics and Astronomy, The Raymond and Beverly Sackler Faculty of Exact Sciences, \\ Tel Aviv University, Tel Aviv, Israel. \\ E-mail: sadehealzt.tau.ac.il
}

\begin{abstract}
Two finely segmented and very compact calorimeters are foreseen for the instrumentation of the very forward region of the ILC and the CLIC detectors; a luminometer to measure the rate of low angle Bhabha scattering events with a precision better than $10^{-3}$ and, at lower polar angles, a detector for fast luminosity estimate. Both devices will improve the hermeticity of the detector in searches for new particles. Due to the expected high occupancy, fast front-end electronics is needed. The calorimeter closest to the beampipe will be hit by a large amount of beamstrahlung remnants, so that radiation hard sensors are required. Results on the performance of the sensor and readout electronics prototypes are summarized.
\end{abstract}

35th International Conference of High Energy Physics

July 22-28, 2010

Paris, France

${ }^{*}$ Speaker.

${ }^{\dagger}$ On behalf of the FCAL Collaboration [1]. 


\section{Introduction}

Two calorimeters are foreseen in the very forward region for the two proposed $e^{+} e^{-}$linear colliders, the ILC and CLIC ${ }^{1}$; LumiCal will be used to determine the luminosity by measuring the rates of Bhabha scattering events, and BeamCal will provide a fast estimate of the luminosity and control of beam parameters by measuring beamstrahlung radiation. Both are designed as cylindrical sensor-tungsten sandwich electromagnetic (EM) calorimeters, each consisting of 30 absorber disks of $3.5 \mathrm{~mm}$ thickness interspersed with sensors. Sensor layers are segmented radially and azimuthally into pads, and front-end (FE) ASICs are positioned at the outer radii.

In order to reach the required accuracy for measurement of the luminosity (better than $10^{-3}$ for most ILC physics channels and $\sim 10^{-4}$ for the GigaZ program), LumiCal sensors must be finely granulated. Regarding BeamCal, the main challenge is the development of radiation hard sensors, due to the high radiation dose expected at low polar angles. In addition, all detectors in the very forward region have to tackle relatively high occupancy, requiring special FE electronics.

\section{Sensor Development and Front-End Electronics Design}

Prototypes of LumiCal sensors, shaped as ring segments of $30^{\circ}$, have been designed and then manufactured by Hamamatsu Photonics (see Fig. 1a). The thickness of the n-type silicon bulk is $320 \mu \mathrm{m}$. The pitch of the concentric $\mathrm{p}^{+}$pads is $1.8 \mathrm{~mm}$ and the gap between two pads is $0.1 \mathrm{~mm}$.

So far polycrystalline CVD diamond sensors of $1 \mathrm{~cm}^{2}$ size and larger sectors of GaAs pad sensors (see Fig. 1b) have been studied for BeamCal. Since large area CVD diamond sensors are extremely expensive, they may be used only at the innermost part of BeamCal. The charge collection efficiency, CCE, was measured for several GaAs batches with different concentrations of dopants, which were irradiated by up to $1.5 \mathrm{MGy}$ (see Fig. 1c). The leakage current of a pad at room temperature before irradiation was about $500 \mathrm{nA}$ at an applied voltage of $100 \mathrm{~V}$. After the irradiation the leakage current approached $1 \mu \mathrm{A}$. For polycrystalline diamond sensor samples the linearity of the response, the leakage current and the signal collection efficiency have been investigated as well. The signal size depends linearly on the number of charged particles crossing the sensors for up to $5 \times 10^{6}$ particles in $10 \mathrm{~ns}$. The leakage current at room temperature depends only slightly on the absorbed dose up to $7 \mathrm{MGy}$. The CCE rises by a factor of two for doses between 0.5 to $1 \mathrm{MGy}$, then drops smoothly approaching the respective value of a non-irradiated sensor.

The FE ASIC of LumiCal is designed to work in two modes, the physics mode and the calibration mode. In the physics mode, EM showers will be measured with large energy depositions on the pads, processing signals of up to $6 \mathrm{pC}$ per channel. In the calibration mode, signals from minimum ionizing particles will be measured, with signals above $2 \mathrm{fC}$. The signal will be reconstructed using at least 8 bit precision. The FE architecture comprises a charge sensitive amplifier, a pole-zero cancellation circuit and a shaper, as shown in Fig. 2a. The LumiCal ADC was designed using a 1.5-bit per stage pipeline architecture with power switching. Test results of the ADC linearity for the prototype show good linearity, and a signal-to-noise ratio of about $58 \mathrm{~dB}$ is obtained in the frequency range up to almost $25 \mathrm{MHz}$.

\footnotetext{
${ }^{1}$ This report summarizes R\&D work performed by the FCAL collaboration [1]. Please see [2] for further details and references.
} 


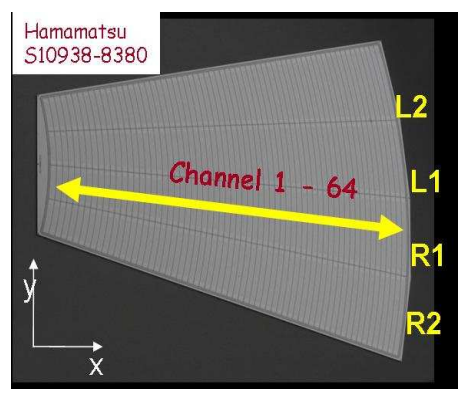

(a)

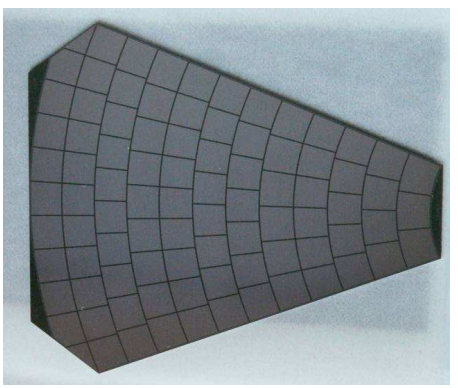

(b)

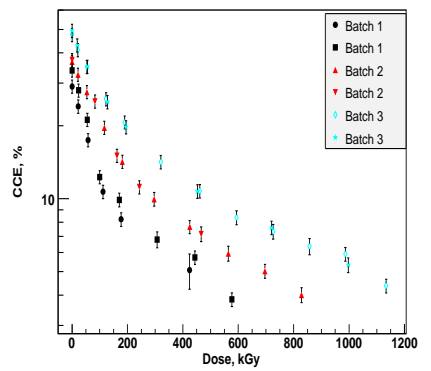

(c)

Figure 1: Prototype silicon sensors for LumiCal (a) and GaAs sensors for BeamCal (b); the CCE as a function of the absorbed dose for the GaAs sensors with different donor concentrations (c). The donor is tellur for batches 1 and 2 and tin for batch 3 .

The BeamCal ASIC, designed for $180 \mathrm{~nm}$ TSMC technology, will be able to handle 32 channels. The two modes of operation require a FE circuit capable of a wide performance envelope; high slew rate for standard data taking, and low noise for calibration. A digital memory array will be used to store the data from all collisions in each bunch train, requiring a sampling rate of 3.25 MHz per channel. This is achieved by 10-bits successive approximation analog-to-digital converters, using one converter per channel. The filter for calibration operation has been implemented using switched-capacitor circuits. In standard data taking operation, an adequate noise power is effectively achieved by means of a slow reset-release technique. Test results of the ADC prototype show good linearity and are consistent with unit capacitance, matching better than $0.1 \%$.

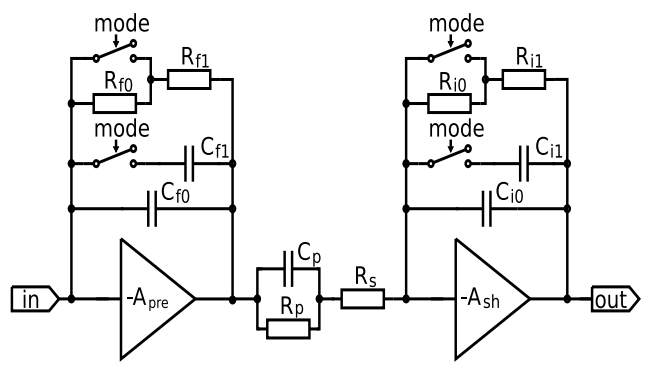

(a)

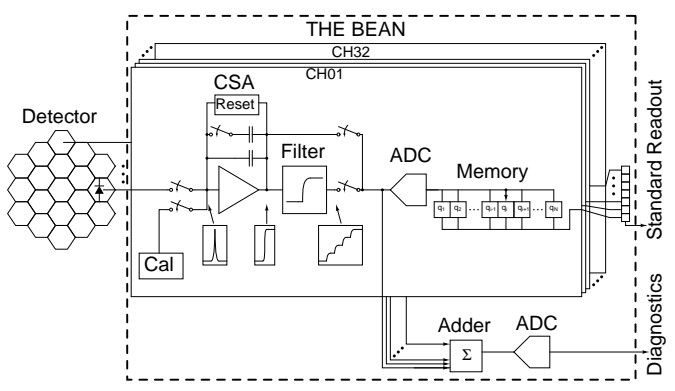

(b)

Figure 2: Block diagram of a single FE channel for LumiCal (a) and for BeamCal (b).

\section{References}

[1] The FCAL Collaboration URL: http://www-zeuthen. desy.de/ILC/fcal/.

[2] H. Abramowicz et al., "Forward Instrumentation for ILC Detectors," ArXiv e-prints, Sept. 2010. URL: http://arxiv.org/abs/1009.2433, Submitted for publication in JINST. 